\title{
Médiévales
}

Langues, Textes, Histoire

61 | automne 2011

La chair des émotions

\section{Amours, castration et miracle au couvent de Watton : évaluation émotionnelle d'un crime d'honneur monastique (v. 1165)}

Love, castration and miracle in the convent of Watton: emotional evaluation of a monastic honor crime (ca 1165)

\section{Damien Boquet}

\section{OpenEdition}

Journals

Édition électronique

URL : https://journals.openedition.org/medievales/6286

DOI : 10.4000/medievales. 6286

ISSN : $1777-5892$

\section{Éditeur}

Presses universitaires de Vincennes

\section{Édition imprimée}

Date de publication : 20 décembre 2011

Pagination : 77-95

ISBN : 978-2-84292-337-2

ISSN : 0751-2708

\section{Référence électronique}

Damien Boquet, «Amours, castration et miracle au couvent de Watton : évaluation émotionnelle d'un crime d'honneur monastique (v. 1165) », Médiévales [En ligne], 61 | automne 2011, mis en ligne le 17 janvier 2012, consulté le 24 avril 2022. URL : http://journals.openedition.org/medievales/6286 ; DOI : https://doi.org/10.4000/medievales.6286 
Médiévales 61, automne 2011, p. 77-96

Damien BOQUET

\section{AMOURS, CASTRATION ET MIRACLE AU COUVENT DE WATTON : ÉVALUATION ÉMOTIONNELLE D'UN CRIME D'HONNEUR MONASTIQUE (V. 1165)}

Au milieu des années 1160, Gilbert de Sempringham (1189) se rendit au monastère cistercien de Rievaulx dans le Yorkshire pour demander à son ami l'abbé Aelred (1167) de l'aider à mettre fin à un scandale qui ébranlait le couvent de Watton, situé non loin de là. À son retour de Watton, Aelred écrivit une longue lettre à un destinataire lointain et anonyme dans laquelle il exposait la série des événements et leur dénouement miraculeux ${ }^{1}$.

Henri Murdac, au temps où il était archevêque d'York - entre 1147 et 1153 - avait confié une fillette âgée de quatre ans à Watton, monastère double fondé vers 1150 qui accueillait, selon les principes d'organisation voulus par Gilbert de Sempringham, une communauté de nonnes et une communauté de chanoines, ainsi que des sœurs et des frères convers. Parvenue à l'adolescence, la jeune oblate s'éprit d'un frère et les deux amants nouèrent quelque temps une idylle secrète. Apprenant qu'elle était enceinte, le frère s'enfuit et le scandale devint public. Les nonnes furieuses molestèrent la sœur coupable et la maintinrent enchaînée dans une cellule. Lorsqu'elles se rendirent compte que la jeune fille était enceinte, elles redoublèrent de mauvais traitements si bien, dit Aelred, qu'on craignit pour la vie du fotus. Gilbert, considérant probablement que seul le châtiment du coupable pouvait mettre un terme au trouble des sœurs, imagina alors un stratagème. Ayant appris qu'un lieu de rendez-vous avait été convenu entre les deux amants, il y envoya plusieurs moines du couvent dont l'un travesti pour tromper la méfiance du fuyard. Le frère fut capturé, roué de coups puis ramené au monastère où il fut livré à la vindicte des nonnes. Celles-ci

1. De sanctimoniali de Watton, PL 195, col. 789-796. Il existe un unique manuscrit de la lettre (Cambridge, Corpus Christi College, 139, $\mathrm{xII}^{\mathrm{e}}$ siècle, $\mathrm{f}^{\circ} 147 \mathrm{r}^{\circ}-149 \mathrm{v}^{\circ}$ ). 
introduisirent la future mère et la forcèrent à émasculer de ses propres mains son amant avant de lui fourrer les testicules sanguinolents dans la bouche. Le frère mutilé fut rendu à la communauté masculine - il semble avoir survécu - tandis que la pauvre fille était remise dans sa cellule. Alors que les nonnes se préparaient à l'accouchement, l'archevêque défunt apparut en songe deux nuits de suite à la jeune femme: la première, il l'enjoignit à la prière et à la confession, et la seconde, elle vit des femmes, suivies par l'archevêque, emporter son bébé. À son réveil l'enfant avait disparu. Les sœurs effrayées commencèrent par accuser la mère d'avoir tué son enfant mais, après avoir constaté par elles-mêmes l'absence de toute trace physiologique de l'accouchement, elles finirent par croire le récit de la prisonnière. Elles en référèrent alors au chef de la congrégation. Avant qu'il n'arrive à Watton, la recluse bénéficia d'une nouvelle intervention miraculeuse : des envoyés de Dieu vinrent la libérer d'une des deux entraves qui enserraient ses pieds. Au petit matin, les sœurs ne purent que se rendre à l'évidence. Gilbert de Sempringham en appela au témoignage d'Aelred qui, une fois sur place, confirma le miracle et donc le retour en grâce du monastère. Quelque temps après son retour à Rievaulx, Aelred reçut une lettre de Gilbert qui lui apprit que la seconde entrave était tombée elle aussi, ce qu'Aelred interpréta comme le signe que la jeune nonne avait été pardonnée.

Les trois phases de ce récit (la liaison amoureuse, la vengeance et la série d'interventions miraculeuses) forment pour Aelred la matière du «réel» avec laquelle il doit composer ${ }^{2}$. L'abbé hérite d'un événement qui possède sa propre causalité : la découverte de la liaison entre les deux jeunes gens a entraîné la vengeance des nonnes qui a elle-même préparé les conditions du miracle. Lorsque Aelred est sollicité par Gilbert, c'est non seulement pour positionner cette trame événementielle vers l'issue miraculeuse, mais aussi pour lever les accusations inhérentes aux différentes phases du récit. Ainsi, l'abbé de Rievaulx doit se faire l'avocat de trois thèses. En premier lieu, toutes les précautions

2. Sur cet épisode, voir en priorité G. Constable, «Aelred of Rievaulx and the Nun of Watton: An Episode in the Early History of the Gilbertine Order», dans D. BAKER éd., Medieval Women, Oxford, 1978, p. 205-226. À compléter par S. Elkins, Holy Women of Twelfth-Century England, Chapell Hill-Londres, 1988, p. 106-111 ; B. Golding, Gilbert of Sempringham and the Gilbertine Order, ca 1130-ca 1300, Oxford, 1995, p. 33-38; E. FreEMAn, «Nuns in the Public Sphere: Aelred of Rievaulx's De sanctimoniali de Watton and the Gendering of Authority », Comitatus, 27 (1996), p. 55-80; S. SALIH, «Monstrous Virginity: Framing the Nun of Watton», Mediaevalia, 20 (1997), p. 49-72; P. G. SснміDт, «Die Nonne von Watton: Amor illicitus, Apostasia, Miraculum», dans M. Thumser, A. Wenz-Haubfleisch et P. WiEgand éd., Studien zur Geschichte des Mittelalters. Jürgen Petersohn zum 65. Geburtstag, Stuttgart, 2000, p. 122-128; AelRed of RievaulX, The Lives of the Northern Saints, Kalamazoo, 2006, introduction par M. L. DutTon, p. 20-26, traduction américaine de la lettre par J. P. Freeland, p. 109-122. Traduction française partielle dans J. Boswell, Au bon cœur des inconnus. Les enfants abandonnés de l'Antiquité à la Renaissance, Paris, 1993, p. 320328. 
avaient été prises par Gilbert pour garantir la clôture féminine si bien que ni le chef de la congrégation ni la communauté conventuelle ne peuvent être tenus pour responsables de cette fâcheuse affaire. Ensuite, la castration de l'amant est une vengeance légitime en pareilles circonstances. Enfin, le miracle lève tout soupçon d'infanticide et manifeste le retour en grâce du monastère.

Le propos de cet article est de montrer que les émotions narrativisées qui accompagnent les violences exercées contre les corps des trois victimes (corps de l'amant roué de coups puis mutilé, corps de la nonne exhibé, fouetté et entravé et corps du fotus molesté) sont moins l'écume sulfureuse du scandale que la clé de son apaisement. Pour conduire cette analyse, je procéderai en trois moments qui sont aussi à mes yeux les étapes d'une intégration progressive de la matière émotionnelle dans un questionnement historique global. Après avoir défini les enjeux politiques de cet épisode dans le contexte de crise que traverse l'ordre gilbertin au milieu des années 1160, j’expliciterai les faiblesses de la défense d'Aelred sur les trois thèses qui constituent la trame visible de l'argumentation. Pour autant, l'abbé se donne les moyens de remplir la mission que son ami Gilbert lui a confiée. Je montrerai alors comment Aelred s'appuie sur la rationalité morale des émotions des nonnes pour pallier ces failles et offrir ainsi un scénario à la fois cohérent et légitime à ce qui se présente au départ comme un enchaînement d'événements hautement problématiques.

\section{Les raisons d'un soutien : amitiés personnelles et solidarités monastiques}

Quand Gilbert requiert l'appui d'Aelred, l'affaire initiale a déjà trouvé sa solution: les nonnes ont lavé l'affront et le problème de l'enfant a été résolu par le miracle. Mais le scandale n'est pas éteint pour autant, peut-être même a-t-il été attisé par les circonstances de la vengeance. Plus largement, il convient de rapporter cet épisode dramatique au climat houleux que connaît l'ordre gilbertin au milieu des années 1160. On est alors aux portes d'une grave crise causée par la contestation de frères convers qui dénoncent auprès du pape, en 1164-1165, la tutelle excessive qu'exercent sur eux les communautés de chanoines ainsi qu'un certain nombre de scandales ${ }^{3}$. Alexandre III confie à Thomas Becket (tous deux sont alors exilés en France) le soin d'enquêter. L'archevêque envoie une première lettre à Gilbert en 1165 dans laquelle il lui demande des comptes sur les scandales

3. Sur la révolte des frères convers, voir R. Foreville, «La crise de l'ordre de Sempringham au XII ${ }^{\text {e }}$ siècle. Nouvelle approche du dossier des frères lais », dans R. Allen Brown éd., AngloNorman Studies VI. Proceedings of the Battle Conference 1983, Woodbridge, 1984, p. 39-57; R. Foreville et G. KeIr éd., The Book of Saint Gilbert, Oxford, 1987, p. LIV-LXII et B. Golding, Gilbert of Sempringham..., p. 40-51. 
touchant certains couvents ${ }^{4}$. Il semble que Gilbert n'ait jamais reçu cette missive. Une seconde suivra en $1166^{5}$ avant qu'une enquête soit officiellement diligentée par Alexandre III de retour à Rome. Nul doute que l'affaire de Watton fasse partie de ces scandales dénoncés par les frères convers et relayés par Thomas Becket. Dans ce contexte, on peut envisager que le destinataire lointain d'Aelred (longe ab his partibus remoto maxime) soit Thomas Becket lui-même, alors réfugié au monastère cistercien de Pontigny ${ }^{6}$. Dès 1166, l'évêque de Norwich Guillaume, qui mène l'enquête pontificale pour les maisons doubles du diocèse de Lincoln, se fait l'écho à son tour des scandales qui ont résulté de la promiscuité entre les chanoines et les moniales dans l'église ${ }^{7}$. Il recommande que les frères convers n'assistent plus aux matines dans l'église des moniales et que seuls deux ou trois chanoines assurent les messes solennelles. À la même période, en 1166-1167, l'archevêque d'York, Roger de Pont-l'Évêque, dirige l'enquête sur Watton: il ne signale alors aucun scandale mais réitère l'injonction de séparation des chanoines et des nonnes ${ }^{8}$. Ces réticences ne sont pas nouvelles. Depuis longtemps déjà, les cisterciens faisaient preuve de défiance à l'encontre des monastères doubles. En 1147, le chapitre général de Cîteaux avait rejeté la requête de Gilbert demandant à ce que des moines cisterciens encadrent la vie religieuse des moniales. C'est d'ailleurs à la suite de ce refus qu'il avait décidé d'adjoindre des communautés de chanoines pour administrer les couvents féminins.

Pourquoi Aelred accepte-t-il de prendre la défense d'un monastère dont l'organisation même allait à rebours des choix cisterciens ? En premier lieu parce qu'il reste fidèle à ses amitiés, y compris au-delà de la mort pour ce qui concerne Henri Murdac, ancien abbé de Fountains qu'Aelred avait déjà soutenu au temps de son élection controversée au siège archiépiscopal d'York en 1147. Surtout, il y a la fidélité qui le lie à Gilbert de Sempringham. Les deux hommes se sont probablement connus dans le Lincolnshire entre 1143 et 1147, quand Aelred était abbé de Revesby, une fondation de Rievaulx située non loin de Sempringham et

4. The Book of Saint Gilbert, p. 346: «Pervenerunt autem ad aures domini pape, pervenerunt et ad nos, quedam maxima scandala, que et ab ordine, et a domibus ordinis vestri, oborta sunt, que etiam per maximam orbis partem ignominiose sunt divulgata.»

5. Ces deux lettres sont éditées en appendice dans The Book of Saint Gilbert, p. 346-348.

6. Ce qui conduit à dater la lettre de 1165-1166.

7. The Book of Saint Gilbert, p. 136-138: «Quod autem sanctitati vestre suggestum audivimus, canonicos cum monialibus in una ecclesia commorari, et inde plurima scandala suboriri...»

8. Voir les deux lettres de Roger, datées de 1166-1167, dans The Book of Saint Gilbert, p. 150152. Comme en écho au plaidoyer d'Aelred, Roger insiste sur la bonne réputation de Watton: «ut fama publica est, honeste habitant» (p. 150). Roger ajoute que, contrairement aux accusations, Gilbert n'a fait emprisonner aucun frère à Watton. S'il fait référence à la présente affaire, stricto sensu, il a raison, puisque c'est une moniale qui a été emprisonnée et que le frère convers a subi un autre châtiment que la prison. 
de Haverholme ${ }^{9}$, à moins que leur rencontre ne soit plus ancienne encore ${ }^{10}$. Dans un sermon, Aelred loue Gilbert mais aussi les nonnes de son ordre, peut-être celles de Watton, pour les grâces de leur chasteté (pudicitia) ${ }^{11}$. En outre, depuis sa fondation, Watton a conservé des liens étroits avec les cisterciens du Yorkshire: avec Rievaulx mais aussi avec Meaux, une fondation de Fountains, située non loin de Watton. En 1160, le premier abbé de Meaux, Adam, résigna sa charge et se fit reclus à Watton durant sept années, ce qui signifie qu'il était probablement sur place au moment où Aelred visita le couvent ${ }^{12}$. En outre, ce sont les mêmes familles aristocratiques qui font des donations à ces différents monastères du Yorkshire. Le fondateur laïc de Watton, Eustache FitzJohn, était très proche de Walter Espec, premier bienfaiteur de Rievaulx. Son fils, Guillaume de Vescy, fait lui-même partie des bienfaiteurs de Rievaulx tandis que le protecteur du monastère cistercien au début des années 1160 , Robert $I^{\text {er }}$ de Ros, se retrouve témoin d'une donation à Meaux et de la confirmation des biens de Watton par Guillaume de Vescy ${ }^{13}$.

Ainsi, il existe au milieu du XII siècle, au-delà des tensions et des divergences, une grande proximité entre ces communautés féminines et le monachisme cistercien, surtout dans le Yorkshire où les couvents gilbertins s'inspirent d'emblée des pratiques cisterciennes ${ }^{14}$. En accédant à la requête de Gilbert de Sempringham, d'abord en se rendant sur place pour constater publiquement le miracle puis en rédigeant cette lettre-plaidoyer, Aelred assume ses obligations, sur un plan personnel par amitié pour Henri Murdac et Gilbert, et sur un plan collectif comme abbé d'un grand monastère qui vit en communauté de destin avec un couvent féminin comme Watton.

Cependant certains indices permettent de comprendre qu'Aelred éprouve gêne et réticences dans ce dossier.

9. B. Golding, Gilbert of Sempringham ..., p. 85.

10. G. Constable, «Aelred of Rievaulx...», p. 210.

11. «Homélie 2 Sur les fardeaux d'Isaïe», dans Aelredi Rievallensis Homeliae de oneribus propheticis Isaiae, G. RACITI éd., Turnhout, 2005 (Corpus Christianorum Continuatio Mediaevalis 2D), p. 36: «Scio in monasteriis virginum, quae sub venerabili et cum summa reverentia nominando sancto Patre Gilleberto uberes pudicitiae fructus cotidie transmittunt.» Cet ensemble de 31 homélies a connu sa mise en forme définitive entre le printemps 1163 et le printemps 1164, à un moment donc où les ennuis ont sans doute déjà commencé pour Gilbert de Sempringham.

12. Voir E. A. Bond éd., Chronica monasterii de Melsa, Londres, 1866, vol. I, p. 107.

13. Voir E. Jamroziak, Rievaulx Abbey and its Social Context, 1132-1300. Memory, Locality, and Networks, Turnhout, 2005, p. 45 (note 93), 87-88 et 158.

14. Les moniales de Haverholme suivent les coutumes cisterciennes tandis que les usages des convers cisterciens ont servi de modèle aux frères et sœurs laïcs de l'ordre gilbertin. Voir A. GRÉLoIs, «Homme et femme il les créa»: l'ordre cistercien et ses religieuses des origines au milieu du XIV siècle, thèse de doctorat inédite, Paris IV-Sorbonne, 2003, p. 211-212. 


\section{Une défense embarrassée}

\section{La clôture féminine}

La question de la clôture des nonnes est au cœur des critiques que subit le monastère. Or, le sujet est important aussi pour Aelred qui en fait l'un des thèmes de son traité $\mathrm{La}$ Vie de recluse ${ }^{15}$ écrit à la même période. Certes, il n'est pas surprenant que dans un texte consacré à la réclusion l'abbé fasse l'apologie d'une clôture stricte. Pour autant, dans cet écrit il livre de façon plus large sa perception des dangers qui menacent les femmes, et les religieuses en particulier, au premier rang desquels on retrouve cette vulnérabilité aux désirs et aux tentations venues du monde extérieur. Les contacts sensoriels sont autant de dangers, par la vue, l'ouïe, le toucher. Tous les sens en matière de désir ont en réalité une propriété tactile qu'Aelred illustre souvent par l'image du venin ${ }^{16}$. La voix aussi est une menace: «Entendre trop souvent la même voix d'homme ne va pas sans danger ${ }^{17}$.» En écrivant ces mots Aelred se souvient-il que les sœurs de Watton, quoique séparées des hommes par une cloison dans l'église, entendaient quotidiennement les chants masculins aux offices ${ }^{18}$ ? On peut le penser en lisant cette plainte qu'Aelred émet à propos des recluses, mais qui raisonne d'un écho particulier si l'on songe à l'affaire de Watton: «De nos jours, c'est déjà bien beau si [les recluses] gardent l'intégrité corporelle, si une grossesse ne vient pas mettre fin à leur réclusion, si des pleurs d'enfants ne trahissent pas qu'elles sont devenues mères ${ }^{19}$ ! » La lecture de La Vie de recluse ne laisse place à aucune ambiguïté : Aelred est favorable à une clôture stricte des religieuses, pas uniquement parce que cet écrit concerne la réclusion volontaire mais aussi parce qu'il concerne les femmes qui ont pour bien le plus précieux leur virginité et qui sont par nature faibles face à la tentation ( «Souviens-toi toujours combien est précieux le trésor que tu portes, et combien fragile l'écrin! Pense à la récompense, à la gloire, à la couronne que te vaudra cette virginité gardée. Et d'autre part, songe sans cesse à la confusion, à la peine, au châtiment qui t'attendent si tu la perds ${ }^{20} \gg$ ).

15. Aelred de Rievaulx, La Vie de recluse, Paris, 1961 (Sources Chrétiennes, 76).

16. Ibid., p. 50 et De sanctimoniali de Watton, col. 791.

17. Ibid., p. 59: «Nam eamdem viri vocem saepe admittere, quibusdam periculosum esse non dubito.»

18. The Book of Saint Gilbert, p. 46.

19. Aelred de Rievaulx, La Vie de recluse, p. 56: «Sufficunt illis quae modo sunt si hanc corporalem castitatem conservent, si non onusto ventre extrahantur, si non fletus infantis partum prodiderit.»

20. Ibid., p. 81 : «Cogita semper quam pretiosum thesaurum in quam fragile portas vasculo, et quam mercedem, quam gloriam, quam coronam, virginitas servata ministret; quam insuper poenam, quam confusionem, quam damnationem importet amissa, indesinenter animo revolve.» 
On a donc un premier point délicat pour Aelred. S'il loue la vertu collective des sœurs et la piété intense de certaines d'entre elles, il se garde bien dans son récit de légitimer la cohabitation des sexes sur un même site conventuel. Et pour cause, c'est qu'il n'y est pas favorable. Seule sa bienveillance envers Gilbert, dont la position est de surcroît en cette période déjà suffisamment délicate, l'empêche de le dire explicitement, du moins dans ce texte.

\section{Les vierges castratrices}

Le châtiment de castration pour adultère ou rapt d'une vierge était pratiqué dans l'Angleterre du XII ${ }^{\mathrm{e}}$ siècle, et ailleurs en Occident malgré la diffusion du droit romain qui prohibait ce genre de pratique mutilatrice ${ }^{21}$. Dans l'Europe du haut Moyen Âge, la castration faisait partie des châtiments dans plusieurs codes de lois pour différents forfaits: dans la loi salique c'est la peine infligée aux esclaves reconnus coupables de vol ou de fornication, dans la loi wisigothique, elle s'applique aux personnes qui s'adonnent à des pratiques homosexuelles ${ }^{22}$. Quant à l'adultère, il peut être puni de mort comme l'attestent plusieurs lois barbares ${ }^{23}$ et de nombreuses sources narratives et juridiques, y compris dans l'Angleterre des Plantagenêts ${ }^{24}$. Au XIII siècle, le juriste anglais Bracton, dans son célèbre traité De legibus et consuetudinibus angliae, préconise toujours le châtiment de castration en cas de rapt et de viol d'une vierge ${ }^{25}$.

Le châtiment infligé à l'amant doit être évalué au regard de ces éléments. La punition est conforme à la nature de la faute et ne saurait en soi être source d'indignation. Même lorsque certaines nonnes demandent à ce que la fille soit brûlée, elles ne font que reprendre dans leur colère un slogan lu ou entendu ailleurs. Ainsi Grégoire de Tours fait état de la mise à mort par le feu d'une

21. Pour une approche d'ensemble, voir J. A. Brundage, Law, Sex, and Christian Society in Medieval Europe, Chicago, 1990², p. 37, 122-123, 207, 291, 456. À compléter par M.S. Kuefler, "Castration and Eunuchism in the Middle Ages», dans V. L. Bullough et J. A. Brundage éd., Handbook of Medieval Sexuality, New York-Londres, 1996, p. 279-306 (sur l'affaire de Watton, p. 289), et J. Murray, «Sexual Mutilation and Castration Anxiety: A Medieval Perspective», dans M. Kuefler éd., The Boswell Thesis. Essays on Christianity, Social Tolerance, and Homosexuality, Chicago et Londres, 2006, p. 254-272 (sur l'affaire de Watton, p. 262-264).

22. M. S. KUEFLER, «Castration and Eunuchism...», p. 288.

23. Voir J.-P. Poly, Le Chemin des amours barbares. Genèse médiévale de la sexualité européenne, Paris, 2003, p. 153-158.

24. Voir les exemples répertoriés par G. Constable, «Aelred of Rievaulx...», p. 215-216. Analyse complémentaire dans J. MurraY, «Sexual Mutilation... », p. 258-259.

25. Bracton (Henricus de Brattona), De legibus et consuetudinibus angliae, «Appellum de raptu virginum», vol. II, p. 414-415, texte latin édité par G. WoodBine, «Bracton online», http://hls15.law.harvard.edu/bracton/Unframed/Latin/v2/414.htm, consulté le 26 septembre 2010. 
femme accusée d'adultère avec un prêtre ${ }^{26}$. Dans le Roman de Tristan de Béroul, strict contemporain de l'affaire de Watton, le roi Marc apprenant l'adultère d'Iseut et de Tristan fait dresser un bûcher pour les y précipiter tous les deux ${ }^{27}$. Même le principe d'une exécution par la communauté des femmes n'est pas sans précédent. Ainsi, à ce que rapporte le missionnaire anglais Boniface au milieu du VIII ${ }^{\mathrm{e}}$ siècle, dans la Saxe païenne, la femme adultère était fouettée, battue par le groupe des femmes puis poussée au suicide par pendaison, la victime participant ainsi, comme dans le cas de la nonne de Watton, à son propre châtiment ${ }^{28}$.

Pour autant, quel que soit le degré de légitimation de l'acte, les sœurs commettent une série de transgressions qu'Aelred ne saurait valider sans réserve. Ainsi, sur le plan juridictionnel, en mutilant l'amant, les nonnes, qui relèvent du for ecclésiastique, appliquent une peine propre à la justice séculière, lui reconnaissant de fait une primauté de compétence en la matière à une époque où les cours ecclésiastiques anglaises tentent d'étendre leur juridiction à tous les crimes et délits sexuels ${ }^{29}$. Par surcroît, même dans cette configuration, elles prennent un grand risque en se faisant elles-mêmes justice sans passer par la justice du roi. Comment ne pas penser, même si l'exemple concerne le royaume capétien, à la répression qui s'abattit quelques décennies plus tôt sur les agresseurs d'Abélard, eux-mêmes châtrés et aveuglés après avoir été livrés à la justice du roi? Dans le Roman de Tristan, ce sont les sujets du roi Marc qui tentent de le dissuader d'exécuter les amants sans procès: «Sire, vous commettriez une horrible faute s'ils n'étaient point jugés au préalable. Exécutez-les ensuite, sire, par pitié ${ }^{30}$ !» Dans l'Angleterre du XIII ${ }^{\mathrm{e}}$ siècle, où le rapt d'une vierge est une atteinte à la paix royale, celui qui se fait lui-même vengeance risque la peine capitale $^{31}$.

Et si le frère convers était mort des suites de ses blessures? On peut penser qu'alors l'indulgence de la justice, royale ou ecclésiastique, aurait été plus difficile à obtenir. On touche ici à une autre transgression, plus grave encore sans doute aux yeux des détracteurs du couvent, liée à l'interdit qui pèse sur

26. G. Constable, «Aelred of Rievaulx...», p. 214-215. L'Histoire des Francs ne relate pas moins de onze affaires d'adultères, qui se règlent presque toujours dans l'homicide, au plus haut niveau de l'aristocratie entre 579 et 591; voir J.-P. Poly, Le Chemin ..., p. 58-59.

27. BÉroul, Roman de Tristan, dans Tristan et Iseut. Les poèmes français. La saga norroise, textes originaux présentés, traduits et commentés par D. LACRoIX et P. WALTER, Paris, 1989, p. 62-81. Voir aussi Ami et Amile. Chanson de geste, publiée par P. F. Dembowsкi, Paris, 1987, p. 24, v. 728733.

28. Lettre citée par J.-P. PoLy, Le Chemin..., p. 154-155.

29. Pour une synthèse du droit canon chez les décrétistes du XII ${ }^{\mathrm{e}}$ siècle en matière de pratiques sexuelles, voir J. A. BRundage, Law, Sex..., p. 256-324.

30. Béroul, Roman de Tristan, p. 62-63.

31. Bracton, De legibus et consuetudinibus angliae, vol. II, p. 408. 
tous les membres de l'Église de verser le sang par violence. Même lorsque la législation canonique préconise un châtiment corporel en cas d'adultère, celle-ci se limite à des coups de fouet en veillant à ce que le sang ne soit pas versé ${ }^{32}$. Enfin, et c'était déjà le constat de G. Constable, il y a de quoi s'insurger devant la cruauté avec laquelle le châtiment est exécuté puisqu'en l'occurrence les nonnes non seulement forcent la pauvre fille à émasculer son amant (est-ce aussi pour se dédouaner de l'accusation de crime de sang?) mais ensuite elles poussent l'agression jusqu'à lui placer les parties génitales dans la bouche. On est bien ici face à un comportement qui relève de la crudelitas, d'une violence excessive que ne légitime en rien le forfait et que le châtiment n'exigeait pas ${ }^{33}$. Venant de la part de femmes et de nonnes, à ce double titre championnes supposées de la retenue et de la pudeur, cette scène ne peut que susciter la réprobation, quelle que soit la nature de l'offense. Dès lors, déplacer l'évaluation de l'acte depuis le terrain judiciaire vers le registre moral devient un impératif pour Aelred: « Je n'approuve pas l'effusion de sang, mais je loue la grande ferveur des vierges saintes contre l'infamie ${ }^{34}$.» Telles sont les prémices qui vont conduire Aelred à mettre l'évaluation émotionnelle au cœur de la dynamique narrative.

\section{Un modeste miracle}

La disproportion dans l'économie du texte entre l'espace consacré au récit du miracle de libération de la nonne enceinte - qui n'occupe que le dernier quart de la lettre - et celui dévolu au rappel des circonstances préalables confirme, si besoin était, que l'objectif d'Aelred est autant d'assurer le destinataire de l'authenticité du miracle que de l'informer sur le contexte précis du scandale. C'est sans doute pour pallier ce déséquilibre, tout en louant le passé du monachisme féminin à Watton, qu'Aelred relate assez longuement en préambule deux miracles, l'un emprunté à Bède le vénérable ${ }^{35}$, le second étant contemporain.

Quant au «merveilleux miracle», qui donne son titre à la lettre dans le manuscrit de Cambridge, il est d'une nature assez singulière qui conduit plutôt à en relativiser l'efficacité au sein de l'argumentaire développé par Aelred. Une

32. Voir par exemple Bernard de PARMe, Glose ordinaire, X 5.17.4v.pecuniaria poteris, Venise, 1486 ou Henri de Suse (Hostiensis), Summa aurea, lib. 5, tit. De penis raptorum corporum, $\S 1$, f. 245vb-246ra, Venise, 1581 (réimp. Turin, 1965), cités dans J. A. BRundage, Law, Sex..., p. 471.

33. Sur cette notion de crudelitas comme violence illégitime contraire à la justitia et à la clementia, voir l'étude de D. BARAz, Medieval Cruelty. Changing Perceptions, Late Antiquity to the Early Modern Period, Londres, 2003, p. 75 et passim.

34. De sanctimoniali de Watton, col. 794 : «nec probo sanguinis effusionem, sed tantam contra turpitudinem sanctarum virginum aemulationem extollo.»

35. BÈDE, Histoire ecclésiastique, 5, 3, qui le situe en un lieu nommé Wetadun. Voir M. L. Dutton, «A Certain Wonderful Miracle», dans Aelred of RievaulX, The Lives..., p. 110, note 2. 
partie au moins de l'épisode miraculeux a suscité des doutes, les plus terribles qui soient puisque la disparition fait naître un soupçon d'infanticide chez les nonnes. Aelred n'élude pas la question, sans doute parce que le soupçon est déjà public. Cela signifie également que certains ont interprété la seconde vision nocturne de la jeune mère comme une reconnaissance de l'abandon de l'enfant, qui d'ailleurs ne cause aucune critique ${ }^{36}$. Tout se passe comme si les interventions surnaturelles ne faisaient qu' apporter une approbation divine à un règlement bien humain de l'affaire : l'enfant est confié à une nourrice et la mère, après un temps de pénitence (signifié alors par la délivrance des entraves en deux temps), est réintégrée dans la communauté monastique. Dès lors, même s'il n'y a aucune raison de penser qu'Aelred met en doute l'authenticité du miracle, le récit qu'il en donne ne conduit pas à l'évaluer comme un «merveilleux miracle» mais plutôt comme un modeste miracle.

Cette modestie vaut jusque dans la façon dont est décliné dans ce récit un type de miracle appelé à devenir populaire à la fin du Moyen Âge. Les récits de délivrance miraculeuse d'une nonne enceinte sont encore rares dans la première moitié $\mathrm{du} \mathrm{XII}^{\mathrm{e}}$ siècle. Cependant, ils commencent à se diffuser précisément dans des compilations insulaires ${ }^{37}$. Toujours est-il que, dans tous ces exemples précoces, le miracle est accompli par la Vierge ${ }^{38}$. Si rien n'empêche d'envisager que c'est Marie qui officie aussi à Watton, Aelred ne dit mot sur ce point et il apparaît plutôt qu'il fait jouer à l'archevêque le rôle dévolu à la Vierge dans les autres récits contemporains.

Enfin, si l'on admet que la fonction première du miracle est d'apaiser le scandale, il faut signaler une ultime faiblesse, qui ne tient pas cette fois à la qualité intrinsèque de l'épisode mais à la difficulté de l'articuler avec l'autre processus de résolution du scandale, à savoir la vengeance. La disparition miraculeuse de l'enfant et la castration du père sont bien les deux actes qui résolvent, l'un selon une logique purement séculière, l'autre selon une logique purement religieuse,

36. C'est la lecture de John Boswell qui souligne l'ironie de l'histoire de cette jeune fille, ellemême abandonnée enfant au couvent, qui se voit contrainte d'abandonner à son tour son bébé; voir J. Boswell, Au bon cœur..., p. 219.

37. Celles-ci sont récapitulées dans G. Constable, «Aelred of Rievaulx...», p. 212-214, notes 25 à 28. À compléter par R. W. Southern, «The English Origins of the Miracles of the Virgin», Mediaeval and Renaissance Studies, 4 (1958), p. 200-201; R. Mazo KarRas, «The Virgin and the Pregnant Abbess: Miracles and Gender in the Middle Ages», Medieval Perspectives, 2 (1988), p. 112-132; J. Boswell, Au bon cœur des inconnus..., p. 256-257 et 329-331, et H. KJeLlman, La Deuxième Collection anglo-normande des miracles de la Sainte Vierge et son original latin, Paris, 1922, p. 60-61.

38. Voir aussi l'iconographie du Miracle de l'abbesse grosse tirée des Miracles de Notre Dame de Gautier de Coinci dans N. Bériou, J. Berloz et J. Longère éd., Prier au Moyen Âge. Pratiques et expériences ( $V^{e}-X V^{e}$ siècle), Turnhout, 1991, pl. VII. 
le même problème. Comment lier ces deux logiques sachant que la trame du récit fait de la vengeance un préalable à la miséricorde divine ? Et c'est bien là l'enjeu : si le miracle n'exige pas en amont la mutilation de l'amant, comment justifier celle-ci dans l'économie narrative? Et sans cette insertion dans le processus miraculeux, il ne demeure que l'horreur brute d'un crime de sang perpétré par des nonnes...

\section{La résolution émotionnelle du scandale}

\section{Pour l'honneur du Christ}

Aelred réussit le tour de force d'établir un lien organique entre les deux étapes de la résolution du scandale (la castration et le miracle) par l'argument de l'honneur du Christ. De ce point de départ découle toute la trame émotionnelle du récit, laquelle devient à la fois nécessaire et efficace : la vengeance des nonnes sert à laver l'injuria Christi. Pourquoi la faute des amants est-elle une injure faite au Christ? En l'occurrence parce que le Christ tient ici la place de l'époux offensé puisque la faute de la nonne relève de l'adultère (adultera). Dès lors, tant que l'offense n'est pas lavée, aucune miséricorde n'est envisagée, laquelle est la condition sine qua non du miracle. C'est pourquoi le terme misericordia n'apparaît dans le texte qu'après que la vengeance a été consommée.

De plus, puisque toutes les sœurs sont épouses du Christ, l'opprobre de l'adultère qui pèse sur l'une contamine toute la communauté. Aelred, en faisant de ce péché de chair un adultère déshonorant le Christ, parvient à imposer l'idée que la réaction collective des nonnes n'est pas seulement le fait d'une solidarité de corps, laquelle pourrait être jugée comme un facteur aggravant leur forfait, mais bien une exigence imposée à chacune en particulier par son statut religieux, donc pleinement légitime. Cela complique les choses puisque les sœurs doivent rétablir et l'honneur du Christ et le leur propre. Car, dans cette configuration exceptionnelle, les nonnes assument les deux pôles genrés de la fama, l'honneur féminin prenant ici le visage de la pudeur. Les termes latins utilisés par Aelred pour qualifier cette pudeur sont typiques : pudor, pudicitia, virginitas ou encore verecundia. À l'injuria Christi correspond l'injuria virginitatis. C'est au regard de cette sexuation de l'honneur qu'il faut comprendre le passage à l'acte des nonnes: par la castration, elles exécutent la vengeance qui revient en théorie aux membres masculins du groupe lésé. Cette transgression des lois du genre résulte de la spécificité de leur condition d'épouses d'un mari céleste. On notera l'exploit rhétorique que constitue ce renversement: alors qu'on est enclin à considérer que cet acte est d'autant moins supportable qu'il est perpétré par des nonnes, Aelred nous conduit à accepter que si des femmes sont en droit d'exécuter un tel châtiment, alors ce n'est concevable qu'à la condition qu'elles 
soient nonnes, épouses du seul être dont personne ne peut exiger un passage à l'acte. Comme le Christ ne saurait être lié par l'esprit de vengeance, il leur revient collectivement de restaurer l'honneur de l'époux. C'est ainsi qu'il faut comprendre leur démarche auprès des frères. S'il s'était agi seulement de punir le jeune homme, les moines pouvaient très bien exécuter eux-mêmes le châtiment, comme ils avaient commencé à le faire en le rouant de coups. Mais, au XII ${ }^{\mathrm{e}}$ siècle, la morale coutumière de l'honneur en cas d'adultère de l'épouse demande à ce que la punition soit infligée par l'époux et ses hommes. Comme il n'est pas pensable d'exiger vengeance du Christ lui-même, ce sont les nonnes-épouses qui l'assurent en son nom. Si les nonnes de Watton ont malgré tout besoin des hommes, c'est uniquement en raison de l'impératif de la clôture, par ailleurs enjeu majeur de l'affaire, et pas du tout, ou si peu, pour exécuter la sentence. Ce crime d'honneur est une affaire de femmes car ce sont bien les femmes qui sont exposées en première ligne. Aelred l'a bien compris et concentre tous ses efforts pour les défendre elles, laissant les chanoines et les convers à l'état d'acteurs falots, simples hommes de main sans émotions ni états d'âme.

\section{Éloge de la jalousie}

Aelred est face à un hiatus. D'un côté, il y a la double offense (injuria) à l'honneur du Christ et à celui du couvent. De l'autre, il y a la mutilation, l'un des châtiments d'honneur admis pour pareille offense. Comme il ne peut approuver le passage à l'acte (ce qu'il appelle le factum), non en raison de la nature de l'acte mais du statut de celles qui le commettent, il va contourner la difficulté en doublant la causalité factuelle, problématique, d'une causalité psychologique - la seconde ne se substituant pas à la première mais la complétant -, laquelle repose sur les émotions et leur capacité à motiver l'action.

En ce sens, la terminologie qui qualifie l'honneur sexuel des nonnes relève déjà du registre affectif: la pudeur ou la vergogne sont en effet pour les lettrés médiévaux simultanément des vertus et des émotions. Pudor et verecundia peuvent aussi bien qualifier l'émotion de honte, voire une crainte de la honte - Aelred dit des sœurs qu'elles «craignent pour leur pudeur» (timentes pudori) -, que la vertu de modestie ou de retenue ${ }^{39}$. Ainsi, la vergogne féminine est ici conjointement le principe moral qui détermine la réaction des moniales et l'émotion qui va les pousser à agir.

Reprenons alors l'ordre de la narration et l'enchaînement émotion-action. Au moment où les nonnes découvrent le forfait de leur consœur, elles éprouvent

39. Voir D. Boquet, «La vergogne historique: éthique d'une émotion sociale», Rives nordméditerranéennes, 31 (2008), p. 7-16 et J.-F. Tномаs, Déshonneur et honte en latin : étude sémantique, Louvain, 2007, p. 323-439. 
un sentiment de sidération (stupor) qui précède la déferlante des émotions et des violences. Dès lors, quatre émotions collectives dominent dans le récit : la crainte (timor), la douleur (dolor, luctus, lamenta), la ferveur (fervor) et surtout l'ardeur religieuse (zelus). Le terme de zelus est utilisé à six reprises par Aelred, dont cinq fois en quelques lignes dans le passage qui fait la transition entre le récit de vengeance et le récit du miracle, alors qu'Aelred s'attache à justifier la réaction des nonnes. Fervor et zelus sont des termes très proches par le sens qui qualifient dans un contexte religieux une expression intense de la piété. En outre, fervor est marqué par une connotation physique évoquant le bouillonnement des passions, tandis que zelus peut renvoyer à une ardeur excessive relevant de la jalousie, d'un attachement immodéré et colérique. Zelus est un terme de l'Ancien Testament, assez polysémique puisqu'il peut désigner l'envie, la jalousie mesquine mais plus souvent la colère divine dans un contexte guerrier. Aelred cite plusieurs exemples tirés de la Genèse et du livre des Nombres, mais le vocabulaire et le contexte bibliques convoqués ici proviennent du livre d'Ézéchiel quand Israël, accusée par Yahvé de lui être infidèle, est comparée à une prostituée vouée à la lapidation ${ }^{40}$. Ainsi le zelus des nonnes est à la fois excessif et admirable: excessif au regard de la modération et admirable par la cause de l'élan. C'est ainsi qu'Aelred résout par l'émotion le paradoxe d'un acte à la fois illégitime et légitime. En outre, il use incidemment de la catégorie grégorienne ${ }^{41}$ de l'ira per zelum qui s'oppose, dans les traités sur les vices et les vertus, à l'ira per vitium ${ }^{42}$. La colère est une émotion ambivalente dans le christianisme dont la valeur est décidée par la fin qu'elle sert. Le zelus est associé à la bonne colère, une émotion vertueuse: l'invoquer comme motivation première des nonnes revient pour

40. Éz. 16, 38-42: «Et judicabo te judiciis adulterarum, et effundentium sanguinem: et dabo te in sanguinem furoris et zeli. Et dabo te in manus eorum, et destruent lupanar tuum, et demolientur prostibulum tuum: et denudabunt te vestimentis tuis, et auferent vasa decoris tui, et derelinquent te nudam, plenamque ignominia: et adducent super te multitudinem, et lapidabunt te lapidibus, et trucidabunt te gladiis suis : et comburent domos tuas igni, et facient in te judicia in oculis mulierum plurimarum: et desines fornicari, et mercedes ultra non dabis: et requiescet indignatio mea in te, et auferetur zelus meus a te: et quiescam, nec irascar amplius.»

41. Voir Grégoire le Grand, Moralia in Job, éd. M. Adriaen, Turnhout, 1979 (Corpus Christianorum Series Latina, 143), lib. 5, par. 45, 1. 176.

42. Voir par exemple Hincmar De Reims, De cavendis et virtutibus exercendis, PL 125, col. 880 ou Thomas de Сноввам, Summa confessorum, éd. F. Broomfield, Louvain, 1968, p. 414: «Ira autem per zelum est quando irascimur contra vitia et contra vitiosos, et possumus optare quod talis ira crescat, quia virtus est.» Cités dans R. E. BARTON, «"Zealous Anger” and the Renegociation of Aristocratic Relationships in Eleventh- and Twelfth-Century France», dans B. H. RosEnweiN éd., Anger's Past. The Social Uses of an Emotion in the Middle Ages, Cornell, 1998, p. 153-170 (ici p. 157). Sur le rapport entre colère juste et mutilations judiciaires, voir, dans le même recueil, l'article de G. BürhER-THIERRY, «"Just Anger" or "Vengeful Anger"? The Punishment of Blinding in the Early Medieval West», p. 75-91. 
Aelred à légitimer l'ira, donc à en neutraliser la dimension négative. Aelred fait l'économie en fait d'une légitimation de la furor des nonnes par des arguments extérieurs à la sphère affective: en nommant leur colère zelus, il impose au lecteur la valeur vertueuse de l'action. L'émotion évalue positivement l'action à venir.

Car c'est aussi par l'argument émotionnel qu'Aelred justifie le passage à l'acte. Le zelus est déjà propension à l'acte au sens où l'ardeur religieuse exige une manifestation. L'émotion motive l'action. Si la cause émotionnelle est légitime, l'action qui en résulte ne peut être totalement condamnable. Ainsi, dans le récit chaque acte de violence est motivé par une stimulation émotionnelle. Lorsque les sœurs apprennent la faute de la jeune fille, elles sont prises d'un élan de fureur: "une ardeur brûlait dans leurs os» (exarsit zelus in ossibus earum) qui les pousse à arracher son voile, symbole justement de la pudeur monastique offensée. Peu après la moniale est dévêtue et fouettée, là aussi sous l'effet de la fervor. Puis c'est la douleur et la crainte d'être humiliées par le scandale qui conduisent les nonnes à molester la jeune fille au point de faire craindre pour la survie du fotus. C'est encore au nom de leur «ardeur pour Dieu» (zelum dei) que les nonnes demandent aux chanoines de leur livrer le frère convers; ce même zelus est enfin la motivation immédiate qui les pousse à forcer l'amante à mutiler son amant.

Attribuer une causalité émotionnelle à l'action n'a rien de surprenant au regard des conceptions anthropologiques du XII ${ }^{\mathrm{e}}$ siècle ${ }^{43}$. Aelred lui-même a décortiqué précisément cette chaîne de l'action dans plusieurs de ses écrits: les émotions (affectus) sont des élans dans l'âme qui prédisposent à l'action, laquelle devient possible lorsque la raison donne son consentement (consensus) et que la volonté transforme l'élan en désir d'action. Aelred précise alors que la valeur morale de l'émotion n'est pas intrinsèque mais dépend de la valeur de la fin visée ${ }^{44}$. Si la fin visée est louable, alors l'émotion est vertueuse. Or, dans ce cas précis, la finalité de l'ardeur qui anime les moniales n'est pas l'acte de castration, qui n'en est que la manifestation contingente. Le zelus des sœurs a pour origine et finalité leur attachement passionné au Christ, lequel ne peut que susciter louange et admiration. Si donc le fait n'est pas louable en raison de sa violence, l'émotion qui le motive l'est: «Je ne loue pas l'acte mais l'ardeur» (non

43. Voir D. Boquet, «Des racines de l'émotion. Les préaffects et le tournant anthropologique du XII ${ }^{e}$ siècle», dans D. BoquET et P. NAGY éd., Le Sujet des émotions au Moyen Âge, Paris, 2009, p. 163-186.

44. Voir par exemple Aelred de Rievaulx, De speculo caritatis, III, 38, dans Opera omnia 1. Opera ascetica, Turnhout, 1971 (Corpus Christianorum Continuatio Mediaevalis, 1), p. 123. Pour une analyse d'ensemble, voir D. Boquet, L'Ordre de l'affect au Moyen Âge. Autour de l'anthropologie affective d'Aelred de Rievaulx, Caen, 2005. 
laudo factum sed zelum) ${ }^{45}$. Ce jugement, qui vient clore le récit de la vengeance, est le point de fuite de toute la construction narrative. En faisant passer la raison des émotions au premier plan, devant l'objectivité de la chaîne des actions, donc des violences corporelles, Aelred s'est forgé l'outil qui lui permet de réévaluer entièrement l'événement. Pour autant, il ne plaide aucunement la folie passagère. C'est bien la valeur morale de l'émotion qui allège la faute inhérente à l'acte. Si l'émotion est vertueuse, alors l'acte qui en résulte, quand bien même il est objectivement répréhensible, ne saurait être un péché. Telle est la conclusion sous-jacente découlant du schéma narratif ${ }^{46}$.

\section{Humiliation et miséricorde}

Dès lors, la castration n'est plus un obstacle au miracle. Mieux encore, la castration définit les conditions du miracle. Aelred prolonge l'efficacité affective de la première phase de son récit jusqu'à en faire un support de l'économie miraculeuse. Soulignons au préalable la rupture dans le lexique des émotions entre les deux dernières phases du récit. Désormais les émotions dominantes ressortissent du registre de la pénitence: timor, dolor, afflictio, confusio. Ces émotions sont celles principalement éprouvées par la jeune moniale, mais la démarche pénitentielle qui suit la vengeance est elle aussi collective.

Dans les quelques lignes qui font la transition entre le récit de la vengeance et le récit du miracle, Aelred établit très clairement une chaîne causale qui va de la vengeance des nonnes à la miséricorde divine en suivant une logique pénitentielle. Afin que cette dynamique fonctionne, deux conditions préalables sont indispensables : la contrition pour la jeune nonne et l'imploration larmoyante de la miséricorde pour ses sœurs. Or, ces deux manifestations émotionnelles suivent directement la mutilation, et ne peuvent s'exprimer qu'à la suite de la mutilation. À chacun sa peine, consécutive au châtiment: la castration a rendu le

45. De sanctimoniali de Watton, col. 794. G. Constable («Aelred of Rievaulx...», p. 208, note 10) remarque que la formule est probablement inspirée de Bernard de Clairvaux dans une célèbre lettre contre les hérétiques rhénans. Bernard juge alors la réaction de la foule qui dressa des bûchers pour y précipiter les accusés: «Nous approuvons l'ardeur mais nous ne conseillons pas l'acte» («Approbamus zelum, sed factum non suademus»). Il reprend ici le vocabulaire de son correspondant Evervin de Steinfeld qui parle cependant avec réprobation de «l'ardeur excessive» (nimio zelo) de la foule. Voir Bernard de Clairvaux, «Sermon 66 sur le Cantique des cantiques», $\S 12$, dans Sermons sur le Cantique, t. 4, Paris, 2003, p. 362 et, pour la lettre d'Evervin, même volume, p. 412-425 (ici p. 416). Sur le sermon 66 et le dossier hérétique rhénan, voir désormais U. BRunN, Des contestataires aux «Cathares»: discours de réforme et propagande antihérétique dans les pays du Rhin et de la Meuse avant l'Inquisition, Paris, 2006, p. 164 sq.

46. Le scénario que je mets au jour peut être comparé à un «script émotionnel» tel que R. A. KASTER le conçoit dans Emotion, Restraint, and Community in Ancient Rome, Oxford, 2005, p. 29 et passim. 
garçon fuyard inapte à la vie conjugale ${ }^{47}$ et la jeune fille a subi une humiliation de sa pudeur. Or, la doctrine du sacrement de pénitence telle qu'elle se dessine à cette même période fait de la honte douloureuse du pécheur (Aelred parle aussi de l'afflictio peccatricis) une condition nécessaire pour être digne de miséricorde. Bien sûr, aux yeux des sœurs, le remords n'a pas suffi : à scandale public, humiliation publique, ainsi que l'exige là aussi la pénitence sacramentelle, même s'il s'agit nécessairement d'une publicité restreinte en raison de l'obligation de clôture. De même que la confusio est nécessaire à l'authenticité du repentir, de même l'imploration collective des sœurs est conditionnée en amont par la vengeance.

La vengeance - par la violence exercée sur les corps - conditionne la libération des émotions pénitentielles qui rendent digne de miséricorde. La miséricorde opère à son tour selon une efficacité émotionnelle puisque la pécheresse émeut le cœur de Jésus (commovebat) en raison même des humiliations qu'elle a endurées (patiebatur). La passivité souffrante de l'âme repentie ébranle le cœur de Jésus: une fois encore, l'émotion motive l'action, de nature divine cette fois.

Ainsi se clôt le scénario émotionnel de l'affaire de la nonne de Watton. Tout avait commencé par une passion charnelle (turpis affectus) qui avait fait offense à la pudeur collective des nonnes. Celles-ci, poussées par leur douleur, la crainte de la honte et leur amour inconditionnel de Dieu (zelus Dei) s'étaient vengées en supprimant l'origine de la faute. Leur ardeur apaisée (tepescente zelo), elles pouvaient implorer (plorant et orant) le Christ afin qu'il préservât leur pudeur virginale (verecundiae virginali). Rejointes dans leurs prières par la jeune nonne chez qui les aiguillons du désir (libidinis incentiva) s'étaient commués en larmes d'affliction (afflictio), elles obtinrent que la miséricorde (misericordia) divine manifestât par le miracle le pardon de la pécheresse et le retour en grâce du monastère. La tempête émotionnelle qui s'était abattue sur Watton, loin d'ajouter à la perturbation des événements, s'était donc révélée, grâce au talent rhétorique d'Aelred, la mieux capable d'en rétablir le cours harmonieux : «tout était sain, tout était pur, tout était beau ${ }^{48}$.»

Les émotions participent de l'appréhension cognitive et morale de notre environnement et des expériences que nous vivons. Même si elles ne relèvent pas du domaine conceptuel, elles nous informent sur nos valeurs et notre niveau de conformation aux normes sociales dans la mesure où le ressenti positif ou négatif nous fait prendre conscience de l'adhésion ou du rejet que nous établissons

47. Sur l'empêchement de mariage pour les castrati, voir J. A. Brundage, Law, Sex..., p. 292, note 153 et p. 456 , note 200 .

48. De sanctimoniali de Watton, col. 195: «sana omnia, munda omnia, pulchra omnia.» 
par rapport à la situation vécue ${ }^{49}$. La répétition de cette sensibilisation peut nous conduire à réviser nos jugements ou bien alors à les conforter ${ }^{50}$. Dans le récit qu'il fait de l'affaire de Watton, Aelred prend un grand soin à expliquer la réaction des nonnes par le biais de l'évaluation émotionnelle qu'elles ont de la situation. La nature de leurs émotions atteste de leur grande valeur morale et, dans le même temps, ces mêmes émotions sont les motivations immédiates de l'action ${ }^{51}$. Aelred doit composer avec une série de violences inacceptables dans un espace conventuel. En d'autres circonstances, il lui aurait suffi d'invoquer la loi de l'honneur. La castration judiciaire n'est pas un acte de passion, elle est même l'inverse puisqu'elle est l'application au nom du roi d'une norme juridique. Mais puisque l'argument judiciaire ici est irrecevable, il ne reste à Aelred que la voie de la légitimation émotionnelle, laquelle offre l'immense avantage d'expliquer le passage à l'acte tout en l'évaluant positivement. Par là, le récit d'Aelred donne à voir une anthropologie affective mais aussi un fonctionnement psychosocial des émotions au sein de cette «communauté émotionnelle ${ }^{52}$ » du couvent de Watton. Jusqu'ici, j'ai pris la précaution de ne pas confondre l'usage rhétorique du registre émotionnel avec les pratiques sociales. Pour autant, la fenêtre sur le vécu peut être entrouverte. En effet, si Aelred veut convaincre, il n'a pas d'autre choix que de construire un scénario qui soit cohérent aux yeux mêmes des détracteurs du couvent, donc qui s'accorde avec les conceptions de ses contemporains en matière de psychologie et d'usage social des émotions. Je ne sais pas si le plaidoyer d'Aelred a convaincu, en revanche je suis sûr qu'il est conforme à ce qui est recevable en matière d'expression publique des émotions, si ce n'est que les «règles culturelles ${ }^{53} »$ que le cistercien suit tacitement ne sauraient

49. Cette théorie selon laquelle les émotions sont des jugements axiologiques vient conjointement de la philosophie et de la psychologie. Voir par exemple, en philosophie, R. Solomon, The Passions, Indianapolis, 1993 et, en psychologie, K. R. Scherer, A. Schorr et T. Johnson éd., Appraisal Processes in Emotion Theory, Methods, Research, Oxford, 2001.

50. Pour une réflexion en ce sens qui s'applique au cadre judiciaire médiéval, voir L. Verdon, «Émotions, justice et normes: la fabrique du lien social», Critique, 716-717 (2007), p. 47-57. L'article s'appuie sur P. Livet, Émotions et rationalité morale, Paris, 2002 et D. L. SMAIL, The Consumption of Justice. Emotions, Publicity, and Legal Culture in Marseille, 1264-1423, Londres, 2003.

51. Sur cette disposition majeure des émotions comme tendance à l'action, voir en priorité N. H. FriJda, The Emotions, Cambridge, 2006.

52. Voir l'article de B. H. Rosenwein dans ce même volume et son désormais classique Emotional Communities in the Early Middle Ages, Londres, 2006.

53. Le concept à succès de «display rules», forgé par Paul Ekman, n'est qu'un habillage culturaliste pour la théorie universaliste des émotions. Voir par exemple P. EкмаN, Emotions Revealed: Recognizing Faces and Feelings to Improve Communication and Emotional Life, New York, 2003 ou P. EKman, «L'expression des émotions », dans B. Rimé et K. Scherer éd., Les Émotions, Lausanne, 1993, p. 183-201. Dans ce même champ d'investigation, on trouve cependant des approches plus ouvertes à la construction sociale, par exemple J. RusseLL, «Is there Universal Recognition of Emotion 
être conçues comme des variations sur une base universaliste puisqu'elles sont en permanence produites, négociées par les contemporains eux-mêmes dans l'interaction de la relation sociale. N'en déplaise à Paul Ekman et aux tenants d'une psychologie réaliste, les «règles culturelles» des expressions émotionnelles sont elles-mêmes construites par ceux auxquels elles sont censées s'appliquer, de même qu'elles sont redéfinies dans chaque configuration intersubjective. Sans en tirer des lois générales sur la psychologie sociale des émotions au XII siècle, l'on apprend de ce «fait divers» tragique que les contemporains d'Aelred vivaient leurs émotions comme des évaluations pratiques et des jugements axiologiques. Quoi qu'on ressente aujourd'hui face aux violences infligées aux corps des trois victimes de cette triste histoire, ce sont bien les émotions les plus passionnelles qui attestent de la rationalité morale des agresseurs et, partant, sauvent l'honneur de la communauté.

Damien Boquet - Université d'Aix-Marseille I, Département d'histoire, 29, avenue Robert Schuman, 13621 Aix-en-Provence CEDEX 1, France

\section{Amours, castration et miracle au couvent de Watton : évaluation émotion- nelle d'un crime d'honneur monastique (v. 1165)}

Vers 1165, le couvent de Watton dans le Yorkshire, fondé par Gilbert de Sempringham, est frappé par un double scandale. Une jeune moniale, qui a noué une idylle avec un chanoine du monastère, tombe enceinte. Furieuses, les sœurs se font livrer le coupable et forcent la future mère à émasculer son amant. L'événement, qui se clôt par une péripétie miraculeuse, prend place au moment où la congrégation gilbertine connaît une crise grave. Il est relaté par l'abbé anglais Aelred de Rievaulx qui intervient à la demande de Gilbert. Le propos de cet article est de montrer comment Aelred s'appuie dans son récit sur la rationalité morale des émotions de la communauté pour légitimer un acte d'une violence inouïe dans un lieu conventuel. Ainsi, les émotions narrativisées, loin d'être l'écume bouillonnante d'un règlement de comptes impulsif, posent les conditions mêmes d'une restauration de l'honneur de la communauté.

Émotions - castration - honneur - Aelred de Rievaulx - Watton.

from Facial Expression? A Review of Cross-Cultural Studies », Psychological Bulletin, 115 (1994), p. 102-141 ou A. TCHERKASSOF, Les Émotions et leurs expressions, Grenoble, 2008. 


\section{Love, castration and miracle in the convent of Watton : emotional evaluation of a monastic honor crime (ca 1165)}

Around 1165, the convent of Watton founded by Gilbert of Sempringham in the Yorkshire was stricken by a double scandal. A young nun, living a love affaire with a canon of the monastery, becomes pregnant. The sisters, furious, get the culprit delivered to them and force the future mother to emasculate her lover. The whole story, which closes by a miraculous incident, takes place when the Gilbertine congregation goes through a serious crisis. We know it by the English abbot Aelred of Rievaulx who intervenes in the affair on request of Gilbert. The aim of this article is to study the way Aelred uses the moral rationality of the emotions of the community in order to legitimize an unprecedented act of violence in a conventual context. Far from being the foam of an impulsive settling of scores, the narrativized emotions recreate the conditions helping to restore the honour of the community.

Emotions - castration - honour - Aelred of Rievaulx - Watton. 
